\title{
Synaptic Activity and Activity-Dependent Competition Regulates Axon Arbor Maturation, Growth Arrest, and Territory in the Retinotectal Projection
}

\author{
Naila Ben Fredj, ${ }^{1}$ Sarah Hammond, ${ }^{1}$ Hideo Otsuna, ${ }^{2}$ Chi-Bin Chien, ${ }^{2}$ Juan Burrone, ${ }^{1}$ and Martin P. Meyer ${ }^{1}$ \\ ${ }^{1}$ Medical Research Council Centre for Developmental Neurobiology, King's College London, London, SE1 1UL, United Kingdom, and ${ }^{2}$ Department of \\ Neurobiology and Anatomy, University of Utah Medical School, Salt Lake City, Utah 84103
}

In the retinotectal projection, synapses guide retinal ganglion cell (RGC) axon arbor growth by promoting branch formation and by selectively stabilizing branches. To ask whether presynaptic function is required for this dual role of synapses, we have suppressed presynaptic function in single RGCs using targeted expression of tetanus toxin light-chain fused to enhanced green fluorescent protein (TeNT-Lc:EGFP). Time-lapse imaging of singly silenced axons as they arborize in the tectum of zebrafish larvae shows that presynaptic function is not required for stabilizing branches or for generating an arbor of appropriate complexity. However, synaptic activity does regulate two distinct aspects of arbor development. First, single silenced axons fail to arrest formation of highly dynamic but short-lived filopodia that are a feature of immature axons. Second, single silenced axons fail to arrest growth of established branches and so occupy significantly larger territories in the tectum than active axons. However, if activity-suppressed axons had neighbors that were also silent, axonal arbors appeared normal in size. A similar reversal in phenotype was observed when single TeNT-Lc:EGFP axons are grown in the presence of the NMDA receptor antagonist MK801 [(+)-5-methyl-10,11- dihydro-5H-dibenzo[a,d]cyclohepten-5,10-imine maleate]. Although expansion of arbor territory is prevented when neighbors are silent, formation of transient filopodia is not. These results suggest that synaptic activity by itself regulates filopodia formation regardless of activity in neighboring cells but that the ability to arrest growth and focusing of axonal arbors in the target is an activity-dependent, competitive process.

\section{Introduction}

Synaptic connections in the visual system are organized such that neighboring retinal ganglion cells (RGCs) project to neighboring targets in the brain. Targeting RGC axons to correct termination zones and restriction of arbor size within the termination zone are crucial for generating such topography. Indeed, excessive axonal arbor growth has been shown to perturb visually guided behavior (Smear et al., 2007). Molecular axon guidance cues and neural activity are both required for generating precise retinotectal connectivity, but the relative contribution that each of these make to axon arbor development is not well understood (Ruthazer and Cline, 2004; Huberman et al., 2008).

Time-lapse imaging studies in Xenopus and zebrafish have shown that development of retinotopic maps is highly dynamic. RGC axon arbor development is characterized by the formation of many short-lived filopodia and nascent synapses, only a fraction of which are maintained in the mature arbor (Alsina et al.,

\footnotetext{
Received March 25, 2010; revised June 21, 2010; accepted June 25, 2010.

This work was supported by Medical Research Council (MRC) Career Development Award G0600107 (M.P.M.), an MRC studentship (S.H.), and National Institutes of Health Grant R01 EY12873 (C.-B.C.). We thank lan Thomson, Matt Grubb, and Andrew Lowe for their comments on this manuscript. Thanks also to Brooke Gaynes for generating the is/2b:GAL4 construct.

Correspondence should be addressed to Martin Meyer, Medical Research Council Centre for Developmental Neurobiology, King's College London, Guy's Hospital Campus, London, SE1 1UL, UK. E-mail: martin.meyer@ kcl.ac.uk.

DOI:10.1523/JNEUROSCI.1556-10.2010

Copyright $\odot 2010$ the authors $\quad$ 0270-6474/10/3010939-13\$15.00/0
}

2001; Meyer and Smith, 2006; Ruthazer et al., 2006; Campbell et al., 2007). Stabilized branches can also increase or decrease in length over the course of a few minutes (Kaethner and Stuermer, 1992; O’Rourke et al., 1994; Rajan et al., 1999; Meyer and Smith, 2006; Ruthazer et al., 2006). Developing arbors then transition from this highly dynamic state to a more stable one; there are far fewer transient filopodia and synapses, and stable branches show relatively little change in length over the course of many hours (Meyer and Smith, 2006). Closure of the dynamic phase of growth is crucial for defining arbor territory and for stable visual system function, but the mechanisms that bring about growth arrest and arbor maturation are not clear. Importantly, synapses selectively stabilize filopodia that then mature into axon branches, and synapses are sites of new filopodia initiation (Alsina et al., 2001; Meyer and Smith, 2006; Ruthazer et al., 2006). Interestingly, filopodia formation occurs preferentially at new synapses, whereas filopodia initiation is suppressed at synapses more than a few hours old (Meyer and Smith, 2006). The mechanism underlying this developmental transition in the ability of synapses to promote filopodia formation is not known. The correlation of synapses with branch stability and branch formation therefore suggests that synaptic transmission at nascent synapses could regulate arbor development by modulating one or both of these processes. To investigate the role of synaptic function during axon arbor growth, we have suppressed presynaptic release of neurotransmitter in single RGCs in zebrafish using targeted expression of tetanus toxin light-chain fused to enhanced green 
fluorescent protein (TeNT-Lc:EGFP). Importantly, this manipulation leaves the electrical state of neurons intact. In the mouse retinotectal system, spontaneous electrical activity in RGCs is needed, independently of synaptic transmission, for the ordering of the retinotopic map and the elimination of exuberant retinal axons (Nicol et al., 2007). Using time-lapse imaging of singly silenced axons, we show that synaptic activity is dispensable for axon branch stability and for generating an arbor of appropriate complexity. However, we show that presynaptic function is required for arresting branch formation events. We also provide evidence that regulation of arbor territory and growth arrest is an activity-dependent, competitive process.

\section{Materials and Methods}

Generation of plasmid constructs and mRNA. 5UAS repeats and a heat shock basal promoter were PCR amplified using the pCASPER plasmid as a template (gift from Dr. Camilla Larsen, Medical Research Council Centre for Developmental Neurobiology, King's College London, UK). A $5^{\prime}$ Ase1 site and $3^{\prime}$ Nhel site were included in the PCR primer sequences. The resulting PCR product was subcloned into the Ase 1 and Nhe1 sites of pEGFP-N2 (Clontech), replacing the cytomegalovirus promoter, thus generating the plasmid 5UAS EGFP-N2. The coding regions of TeNT-Lc and the nonproteolytic mutant form of TeNT-Lc (TeNT-Lc mut) (gift from Dr. Sean Sweeney, University of York, Heslington, York, UK) were PCR amplified with primers that generated a 5' EcoR1 site and kozak sequence and a $3^{\prime}$ triglycine linker and Smal site. The resulting PCR products were cloned into the EcoR1 and Sma1 sites of 5UAS EGFP-N2 to generate TeNT-Lc:EGFP and TeNT-Lc mut:EGFP. For generating mRNA encoding TeNT-Lc:EGFP and TeNT-Lc mut:EGFP, the entire coding region for each fusion protein was excised from TeNT-Lc:EGFP or TeNT-Lc mut:EGFP using Notl and EcoR1. The Notl site was blunted, and the fragments were then subcloned into the EcoR1 and Stu1 sites of the pCS2 + plasmid. The resulting plasmids were used as templates for in vitro transcription of mRNA using the mMessage mMachine kit (Ambion). To generate TeNT-Lc:EGFP Tol2, a fragment that included 5UAS, TeNT-Lc:EGFP, and the simian virus 40 (SV40) polyadenylation signal was excised from TeNT-Lc:EGFP with Ase1 and Stu1. The Ase1 site was blunted and the entire fragment was cloned into the EcoRV site of pminiTol2 (Balciunas et al., 2006). Tandem-dimer tomato (TdT) (Shaner et al., 2004) was PCR amplified with primers encoding a 5' Sma 1 site and $3^{\prime}$ Not1 site. The PCR product was cloned into these sites in 5UAS EGFP-N2 so that EGFP was replaced by TdT to generate 5UAS TdT. To generate a fusion of TeNT-Lc to TdT, the coding region for TeNT-Lc was subcloned into the EcoR1 and Smal sites, which placed TeNT-Lc at the N terminus of TdT. To drive expression of TeNT-Lc:TdT in hippocampal neurons, TeNT-Lc:TdT was subcloned into a vector containing the $\beta$-actin promoter.

Hippocampal cultures and transfection. Dissociated hippocampal cultures were prepared from embryonic day 18 Sprague Dawley rats. Neurons were dissociated using trypsin $\left(5 \mathrm{mg} / \mathrm{ml}\right.$ for $15 \mathrm{~min}$ at $37^{\circ} \mathrm{C}$; Worthington), followed by trituration and then plated onto $18 \mathrm{~mm}$ coverslips coated with poly-D-lysine $(50 \mu \mathrm{g} / \mathrm{ml})$ and laminin $(20 \mu \mathrm{g} / \mathrm{ml})$. Culture media consisted of Neurobasal media, 2\% B27 supplement, 1\% glutamax (Invitrogen), and 1\% penicillin/streptomycin (Sigma). Cultures were maintained at $37^{\circ} \mathrm{C}$ in a humidified incubator with $5 \% \mathrm{CO}_{2}$. Neurons were transfected at day 7 using Lipofectamine 2000 (Invitrogen). All experiments were performed at day 14 in vitro.

Imaging synaptophysin-pHluorin responses. Synaptophysin-pHluorin (sypHy) plasmid was a gift from Dr. L. Lagnado (Laboratory of Molecular Biology, Cambridge, UK). Experiments were done as described previously (Li et al., 2005). Briefly, coverslips were mounted in a custommade chamber equipped with a pair of parallel platinum electrodes $\sim 5$ $\mathrm{mm}$ apart, in HEPES-buffered saline (HBS) containing $0.025 \mathrm{~mm}$ APV and $0.02 \mathrm{~mm}$ CNQX. Neurons were stimulated by delivering a $1 \mathrm{~ms}, 25$ $\mathrm{mA}$ current pulses using an SD9 stimulator (Grass Instruments), whose timing was controlled by a transistor-transistor logic signal from the imaging software (Slidebook). Bafilomycin A (Calbiochem) was used at a final concentration of $1 \mu \mathrm{M}$. Images were obtained using an Olympus
IX71 inverted microscope with a CCD camera (Coolsnap HQ) controlled by Slidebook software (Intelligent Imaging Innovations). The light source was a xenon-arc lamp (Lambda LS; Sutter Instruments), in which light exposure was regulated by a rapid shutter (smartShutter; Sutter Instruments) controlled by a Sutter Instruments lambda 10-3 controller, fitted with a $470 \pm 20 \mathrm{~nm}$ bandpass excitation filter (Chroma Technology Corporation) and suitable neutral density filters. Experiments looking at spontaneous vesicle fusion were performed in a humidified, temperature-controlled chamber set at $37^{\circ} \mathrm{C}$, in a calcium-free HBS containing the following (in mM): 0.001 TTX, 0.025 APV, and 0.02 CNQX. Images were collected using a Nikon A1R confocal microscope using a $488 \mathrm{~nm}$ excitation laser and the appropriate emission filters. Long-term imaging experiments made use of the dynamic auto focus unit to correct for any focus drift during the long periods of acquisition.

FM4-64 staining. Neurons were first washed in HBS (in mм): 139 $\mathrm{NaCl}, 2.5 \mathrm{KCl}, 10 \mathrm{HEPES}, 10$ D-glucose, $2 \mathrm{CaCl}_{2}$, and $1.3 \mathrm{MgCl}_{2}, \mathrm{pH} 7.3$ (290 mOsm). Synaptic boutons were then loaded with $10 \mu \mathrm{M}$ FM4-64 [N-(3-triethylammoniumpropyl)-4-(6-(4-diethylamino)phenyl) hexatrienyl)pyridinium dibromide] (Invitrogen) in the presence of 60 mM KCl solution (in mM: $78.5 \mathrm{NaCl}, 60 \mathrm{KCl}, 10$ D-glucose, $10 \mathrm{HEPES}, 2$ $\mathrm{CaCl}_{2}, 1.3 \mathrm{MgCl}_{2}, 0.001 \mathrm{TTX}, 0.025 \mathrm{APV}$, and $0.02 \mathrm{CNQX}$ ) for $90 \mathrm{~s}$ at room temperature. Finally, the neurons were washed with HBS for 10 min to remove excess FM4-64 and fixed for $30 \mathrm{~min}$ with 4\% paraformaldehyde/0.33 m sucrose. Images of hippocampal cultures were acquired using Slidebook software (Intelligent Imaging Innovations) and a CCD camera mounted on an Olympus IX71 inverted microscope with an oil lens [100×, 1.4 numerical aperture (NA); Olympus]. The filter set used to acquire GFP transfected cells were $470 / 30 \mathrm{~nm}$ bandpass exciter, 515/40 $\mathrm{nm}$ bandpass dichroic, and 510/30 nm bandpass emission. FM4-64 was acquired using 565/44 $\mathrm{nm}$ bandpass exciter, $590 \mathrm{~nm}$ long-pass dichroic and 650/52 nm bandpass. Images were analyzed using custom routines in Matlab (MathWorks) as described previously (Li et al., 2005).

Calcium imaging. Bolus injections of Oregon Green 488 BAPTA-1 AM (Invitrogen) were performed as described previously (Niell and Smith, 2005). For electrical stimulation of the retina/optic nerve, 5-6 d postfertilization (dpf) larvae were anesthetized in $0.02 \%$ Tricaine and mounted in $1.2 \%$ agarose on a microscope slide. A glass microelectrode was inserted into the retina near the optic disc, and a $500 \mathrm{~ms}$ stimulus train consisting of $2 \mathrm{~ms}, 20 \mathrm{~V}$ pulses at $20 \mathrm{~Hz}$ was applied using a Grass S88 stimulator (Grass Instruments). Imaging of Oregon Green 488 fluorescence in the tectum was performed using an LSM 710 confocal microscope equipped with a spectral detection scan head and a $40 \times / 1.0 \mathrm{NA}$ water-immersion objective (Carl Zeiss). Images were acquired at a rate of $10 \mathrm{~Hz}$. Fluorescence data were analyzed using custom Matlab software (MathWorks). To define responding larvae a threshold was set at three times the SD of the baseline fluorescence for each larva analyzed.

Behavioral assays. The optokinetic response assay was performed according to Brockerhoff (2006). Touch responses were elicited by a light touch to the head of larvae. Visual background adaptation was assayed by exposure to room light for $15 \mathrm{~min}$, followed by visual inspection of pigmentation using a stereomicroscope.

Generation of the Isl2b:GAL4 transgenic line. An isl2b.2:GAL4 transgenesis construct was generated by multisite Gateway recombination using the Tol2kit system (Kwan et al., 2007) from entry clones p5Eisl3delta16-22 (isl2b.2 genomic fragment: $17.6 \mathrm{~kb}$ upstream of the start codon, from which repeat sequences have been removed by deleting from -7.2 to $-4.3 \mathrm{~kb}$ ), pME-Gal4VP16[413-470] (galactosidase-4 DNA binding domain fused to partial VP16 transactivation domain), and $\mathrm{p} 3 \mathrm{E}-\operatorname{poly}(\mathrm{A})$ (SV40 late polyadenylation signal) and destination vector pDestTol2CG2 (bears cmlc2:egfp transgenesis marker and Tol2 transposon ends). DNA for this construct was coinjected with Tol2 transposase RNA into one-cell stage embryos, which were raised to adulthood and screened for green hearts to test for germ-line integration.

Microinjection of zebrafish embryos. To generate transient and mosaic expression of transgenes in RGCs, $4 \mathrm{~kb}$ of upstream sequence of the zebrafish atoh7 gene was used to drive expression of GAL4 on an activator plasmid (gift from Prof. Steve Wilson, University College London, London UK) that was coinjected with UAS:TeNT-Lc:EGFP or UAS: TeNT-Lc mut:EGFP. Plasmid DNA was prepared using Qiagen miniprep 
kits, and both effector and activator plasmids were injected at a concentration of $25 \mathrm{ng} / \mu \mathrm{l}$ in Danieau solution into one- to four-cell stage zebrafish embryos. mRNA was diluted in Danieau solution and injected at a concentration of $20 \mathrm{ng} / \mu \mathrm{l}$. Injected embryos were raised at $28^{\circ} \mathrm{C}$, on an 11/13 h light/dark cycle in Danieau solution containing phenylthiourea to block pigment formation. To generate zebrafish expressing TeNT-Lc: EGFP in many RGCs, UAS:TeNT-Lc:EGFP Tol2 and synthetic Tol2 transposase mRNA were coinjected into single-cell embryos from the isl2b:GAL4 transgenic line. To label isolated cells in these fish, 5UAS:TdT was also included in the injection mixture. Both plasmids and transposase mRNA were injected at a concentration of $17 \mathrm{ng} / \mu \mathrm{l}$.

DiI labeling. Zebrafish larvae at $6 \mathrm{dpf}$ were fixed overnight at $4^{\circ} \mathrm{C}$ in $4 \%$ paraformaldehyde in PBS, pH 7.4. Fixed larvae were washed several times in low-strength PBS (regular PBS diluted 1:3 in deionized water). Larvae were then mounted on a microscope slide in $1.2 \%$ low-melting-point agarose made in low-strength PBS. A window was cut in the agarose over the eye to allow access of the injection needle. DiI (Invitrogen) was dissolved in $92 \%$ dimethylformamide $/ 8 \% \mathrm{H}_{2} \mathrm{O}$ at $37.5 \mathrm{mg} / \mathrm{ml}$ and pressure injected via micropipette into a nasodorsal patch of retina. Larvae were left overnight at room temperature to allow transport of DiI along RGC axons. Larvae were imaged $1 \mathrm{~d}$ after injection.

Imaging. Zebrafish larvae from 3-7 dpf were mounted on a microscope slide imaging chamber in $1 \%$ agarose and covered with Danieau solution. The agarose was sufficient to restrain movements of the larvae so that anesthesia was not required. Larvae could be maintained in this configuration for imaging sessions lasting at least $10 \mathrm{~h}$, at the end of which they appeared healthy and continued to develop normally. To block NMDA receptors (NMDARs), MK-801 [(+)-5-methyl-10,11dihydro-5H-dibenzo $[a, d]$ cyclohepten-5,10-imine maleate] was added to the rearing medium at a concentration of $10 \mu \mathrm{M}$. MK-801-containing media was exchanged for fresh media daily. Time-lapse imaging was performed overnight to coincide with the normal light/dark cycle under which larvae were raised. Imaging of EGFP-labeled axons was performed using an Ultima IV two-photon microscope (Prairie Technologies) with a $60 \times / 0.9$ NA water-immersion objective (Olympus). Excitation was provided by a Mai Tai titanium:sapphire femtosecond pulsed laser system (Newport-Spectraphysics) tuned to $920 \mathrm{~nm}$. The emission filter set for imaging EGFP consisted of $575 \mathrm{~nm}$ DCXR dichroic mirror and $525 / 70 \mathrm{~nm}$ barrier filter. Imaging of specimens labeled with DiI and with TeNT-Lc:EGFP and TdT was performed using an LSM 710 confocal microscope equipped with a spectral detection scan head and either a $40 \times / 1.0$ NA or $20 \times / 1.0$ NA water-immersion objective (Carl Zeiss). For all samples, optical sections were obtained at $1.5 \mu \mathrm{m}$ intervals.

Image analysis. Because of the planar nature of RGC axonal arbors, we were able to perform morphological analyses on maximum intensity projections of the entire image volumes. Branch number and arbor area were measured using NIH ImageJ, and branch lengths were measured using the NIH ImageJ plugin NeuronJ. For arbors measured at 3, 5, and $7 \mathrm{dpf}$, axonal processes that were $<1 \mu \mathrm{m}$ in length and/or $<0.5 \mu \mathrm{m}$ in diameter were excluded from analyses because time-lapse analysis suggests that such structures tended to be transient. Because most images were acquired with a similar scale, these smaller axonal processes could be digitally excluded by applying a median filter with a radius of 3 pixels $(\sim 0.69 \mu \mathrm{m})$. Lifetimes of all axonal processes were measured by marking all such processes that appeared during a time-lapse sequence. These were scored for total lifetime using custom Matlab software (MathWorks) (Niell et al., 2004). DiI data was also analyzed using NIH Image J. To analyze the area of the DiI-labeled RGC projection field, a polygon was drawn around the labeled area and measured from the point at which RGC axons defasiculated on entering the tectum. Retinal injection area was measured from a single optical section corresponding to the most broadly labeled region in the RGC layer. Statistical analysis was performed using GraphPad Prism (GraphPad Software). Significance level was set to 0.05 ( $95 \%$ confidence intervals). To test whether values came from a Gaussian distribution, Kolmogorov-Smirnov, and D'Agostino and Pearson's omnibus normality tests were used.

\section{Results}

\section{A fusion of TeNT-Lc to fluorescent proteins suppresses synaptic function}

To suppress synaptic activity in single RGCs, we used targeted expression of TeNT-Lc. TeNT-Lc, which inhibits neurosecretion by cleaving the synaptic vesicle membrane protein VAMP2/ Synaptobrevin-2, has proved to be a powerful tool for genetic manipulation of neural circuits in a wide range of model organisms. To unambiguously identify single RGCs expressing TeNT-Lc in vivo, we fused TeNT-Lc to EGFP. To control for nonspecific effects caused by overexpression, we performed parallel experiments using a nonproteolytic mutant form of TeNT-Lc [which harbors a point mutation at position $234(\mathrm{E} \rightarrow$ Q)] fused to EGFP (TeNT-Lc mut:EGFP) (Galli et al., 1994). Using the genetically encoded reporter of presynaptic activity, sypHy (Granseth et al., 2007), we found that evoked release was completely abolished in dissociated hippocampal neurons expressing TeNT-Lc fused to a red fluorescent protein, Tandemdimer Tomato (Fig. $1 A-C$ ). Stimuli that normally mobilize the rapidly releasable pool of vesicles ( 40 action potentials at $20 \mathrm{~Hz}$ ) caused measurable rises in fluorescence in control neurons but no changes in fluorescence for neurons expressing TeNT-Lc $(\Delta F / F$ : control, $0.20 \pm 0.034$; TeNT-Lc, $0.01 \pm 0.007)$. This loss of evoked release was matched by a dramatic decrease in spontaneous release (Fig. 1D,E). Addition of bafilomycin, a vesicular proton pump antagonist, results in additive increases in fluorescence as vesicles fuse spontaneously with the plasma membrane in the absence of activity (Fig. 1D,E). Control neurons show an exponential rise in fluorescence, with a time constant of $\sim 5 \mathrm{~min}$ (Fig. $1 \mathrm{Di}$, black trace in $E$ ), whereas neurons expressing TeNT-Lc show a much slower rate of release with a time constant of $26 \mathrm{~min}$ (Fig. 1 Dii, blue trace in E). In agreement with this sypHy data, we found that using FM4-64 to label active presynaptic terminals in neurons transfected with TeNT-Lc:EGFP resulted in a 2.5 -fold reduction in FM4-64 fluorescence labeling intensity when compared with control neurons transfected with TeNT-Lc mut:EGFP (Mann-Whitney test, $p<0.001$ ) (Fig. $1 F, G$ ). These results demonstrate that TeNT-Lc retains its proteolytic activity when fused to EGFP. To ensure that TeNT-Lc:EGFP was also functional in vivo, mRNA encoding the fusion protein was injected into singlecell stage zebrafish embryos. This resulted in expression of TeNTLc:EGFP throughout the embryo (visualized by EGFP fluorescence). Widespread expression of TeNT-Lc:EGFP caused paralysis, and two visually evoked behaviors, the optokinetic response and visual background adaptation, were also suppressed by TeNT-Lc:EGFP expression. These results indicate that TeNTLc:EGFP is also functional in vivo (Fig. $2 A, B$ ). Because of the transient nature of expression resulting from mRNA injection, we occasionally saw some of these behaviors return in larvae in which they were previously suppressed (Fig. $2 B$ ). This suggests that sustained suppression of these behaviors (until $7 \mathrm{dpf}$ in most cases) is attributable to the continued suppression of presynaptic function by TeNT-Lc:EGFP rather than to developmental defects caused by early expression of TeNT-Lc:EGFP. To examine synaptic function in RGCs directly, we attempted to perform sypHy experiments in vivo. However, rather than accumulating at presynaptic sites, sypHy (which is based on mouse synaptophysin) is diffusely distributed in RGC axons (supplemental Fig. 1, available at www. jneurosci.org as supplemental material). This is in contrast to a fusion of zebrafish synaptophysin that forms discrete aggregates at presynaptic sites (Meyer and Smith, 2006) (supplemental Fig. 1, available at www.jneurosci.org as supplemental material). For 
A

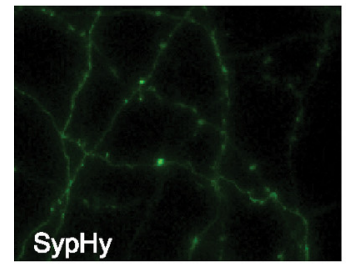

B

i Control

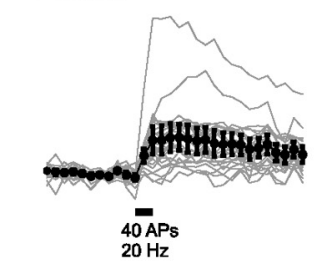

D

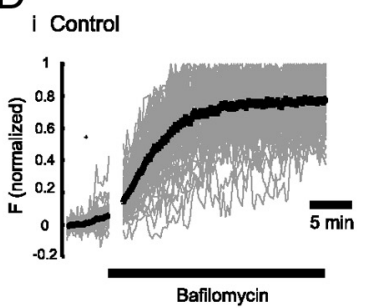

$\mathrm{F}$
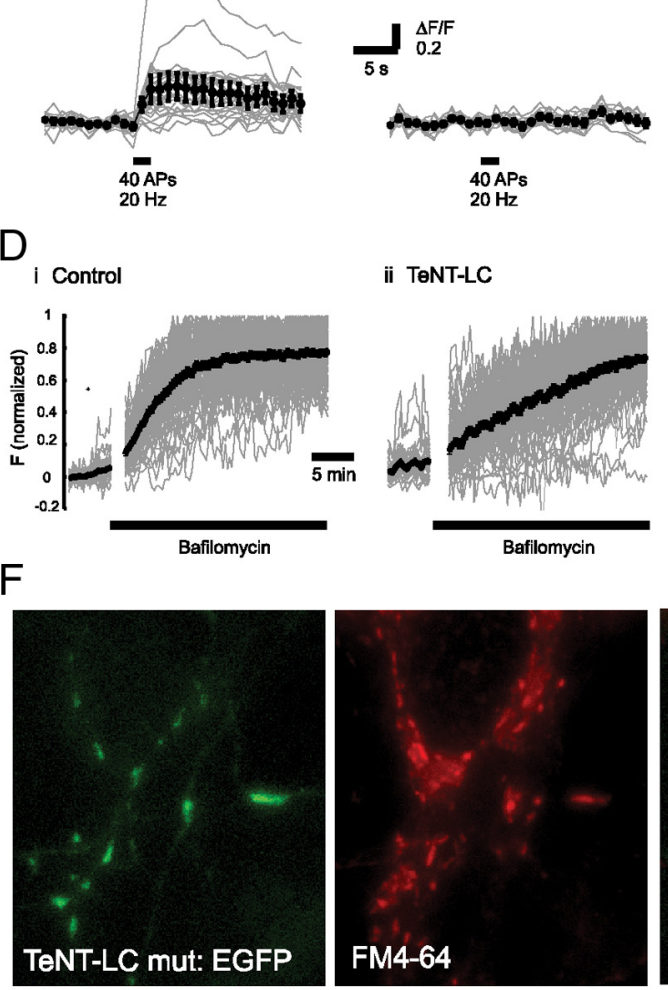

ii TeNT-LC

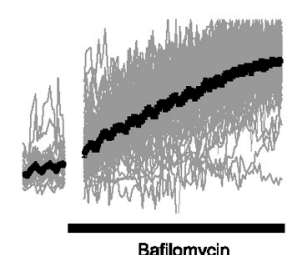

Bafilomycin
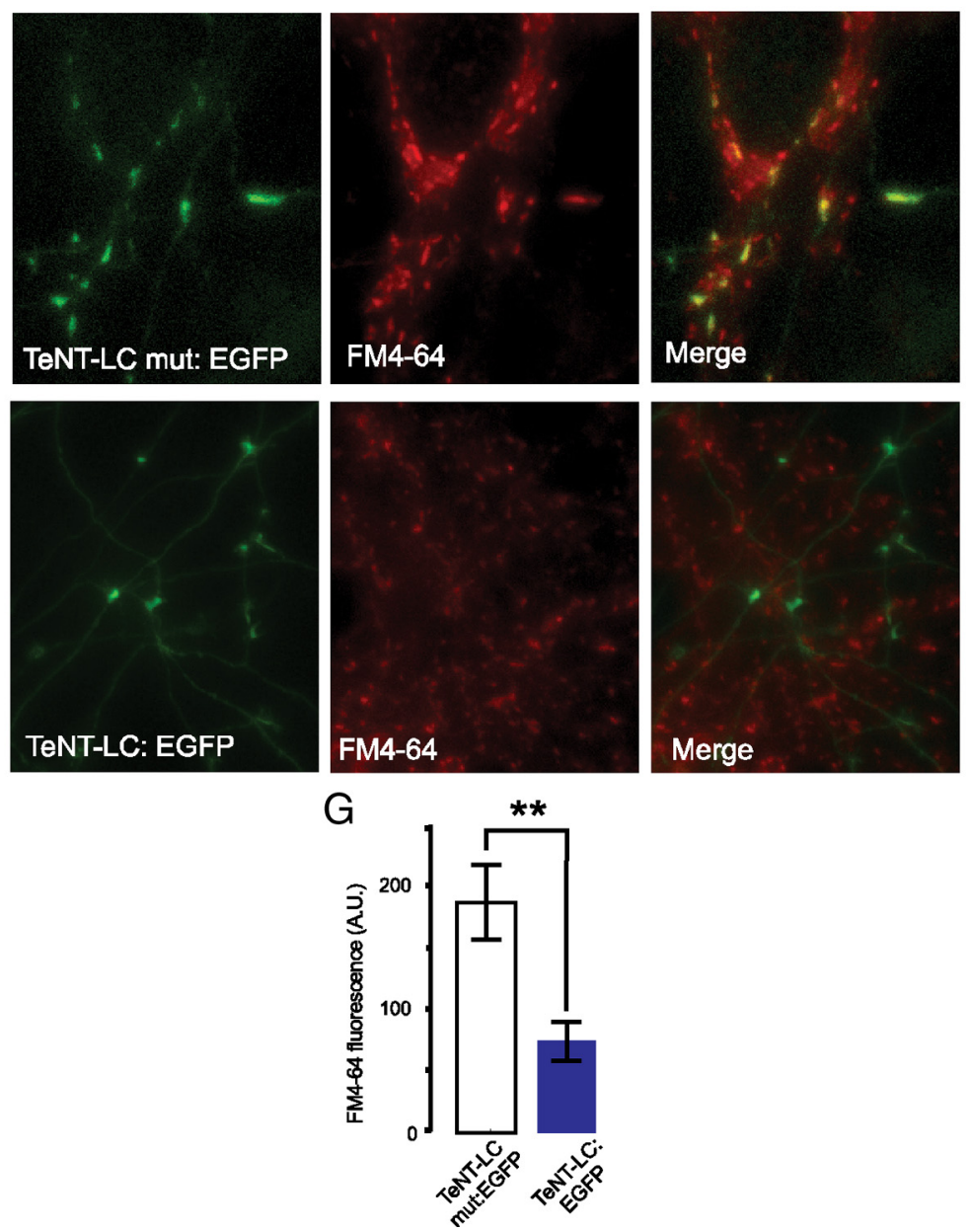

Figure 1. Expression of TeNT-Lc fused to fluorescent proteins blocks neurotransmitter release in hippocampal neurons. $\boldsymbol{A}$, Images of axons expressing sypHy (green) and TeNT-Lc:TdT (red). $\boldsymbol{B}$, Changes in sypHy fluorescence $(\Delta F / F)$ for control (i) and
sypHy to report vesicle function, its fluorescence must be quenched by correct targeting to the lumen of synaptic vesicles at rest. As a likely consequence of its mislocalization, we were unable to observe stimulusevoked fluorescence changes in sypHy expressed in RGCs. As an alternative approach to looking at retinotectal function, we performed functional imaging of tectal cells loaded with the calcium indicator Oregon Green 488 BAPTA-1 AM (Fig. 2C). In $87.5 \%$ of wild-type larvae $(n=8)$, electrical stimulation of the retina elicited reliable and large calcium transients in tectal cells (Fig. 2B-D) (supplemental Movie 1, available at www.jneurosci.org as supplemental material). In contrast, only $27 \%$ of larvae expressing TeNT-Lc:EGFP $(n=11)$ showed any stimulus-evoked responses in tectal cells (Fig. 2B-D). These results suggest TeNTLc-EGFP blocks synaptic transmission in vitro and in vivo and are consistent with previous studies demonstrating that a fusion of TeNT-Lc to cyan fluorescent protein (a closely related derivative of EGFP) blocks synaptic transmission in zebrafish (Asakawa et al., 2008).

\section{Single axons silenced by TeNT-Lc:EGFP have enlarged arbors}

To express TeNT-Lc:EGFP in single RGCs, we used the GAL4-UAS system (Köster and Fraser, 2001). A 4 kb up-

$\leftarrow$

TeNT-Lc-expressing (ii) neurons in response to 40 action potentials at $20 \mathrm{~Hz}$ (black bar). Fluorescence profiles of individual synapses (gray traces) are shown together with the average change in fluorescence (black trace). C, Mean response profile for control neurons (black; $n=5$ ) and neurons expressing tetanus toxin (blue; $n=4$ ). $\boldsymbol{D}$, Spontaneous increases in sypHy fluorescence after addition of bafilomycin (black bar) for control (i) and TeNT-Lc:TdT-expressing (ii) neurons. Fluorescence profiles of individual synapses (gray traces) are shown together with the average change in fluorescence (black trace). Note that the fluorescence is normalized to the maximum value for each trace. $\boldsymbol{E}$, Plot showing an overlay of the increase in fluorescence after bafilomycin treatment for control (black) and TeNT-Lc-expressing (blue) neurons. Bafilomycin was added at time-point zero. Data are the same as that shown in D. Curves were fit with a single-exponential function (red), from which we established the time constant for each condition (control $\tau=4.8 \mathrm{~min}$; tetanus-toxin $\tau=29.5 \mathrm{~min}$ ). $\boldsymbol{F}$, Dissociated rat hippocampal neurons transfected with an inactive mutant form of TeNT (TeNT-Lc mut:EGFP; top row) and the functional form of TeNT-Lc fused to EGFP (TeNT-Lc:EGFP; bottom row). Presynaptic function assayed by FM4-64 labeling is suppressed in neurons expressing TeNT-Lc:EGFP compared with TeNT-Lc mut:EGFP (middle column). The EGFP and FM4-64 images are merged in the right column. Scale bar, 5 $\mu \mathrm{m} . \mathbf{G}$, Quantification of FM4-64 data. Mean values \pm SEM of 209 synapses in 13 TeNT-Lc mut:EGFP-expressing neurons and 113 synapses in 7 TeNT-Lc:EGFP-expressing neurons are shown. Statistical analysis was performed using a MannWhitney test ( $\left.{ }^{* *} p<0.01\right)$. A.U., Arbitrary units. 


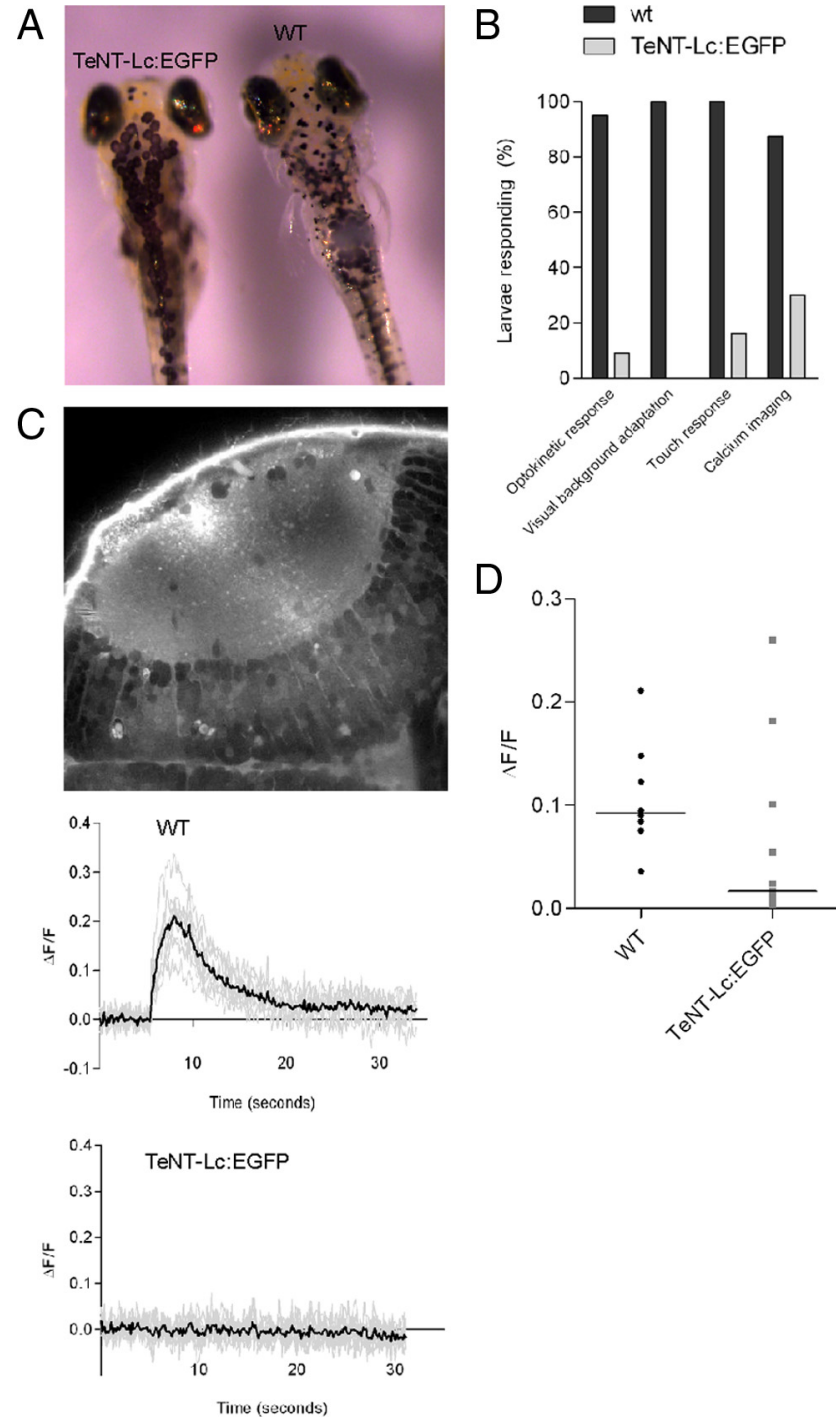

Figure 2. TeNT-Lc:EGFP suppresses synaptic function in vivo. $\boldsymbol{A}$, TeNT-Lc:EGFP expression inhibits visually evoked background adaptation. In bright light, wild-type (WT) larvae $(n=20$; right side) contract black pigmented melanophores in the skin. TeNT-Lc:EGFP-expressing larvae ( $n=37$; left side) fail to contract melanophores in bright light. $\boldsymbol{B}$, TeNT-Lc:EGFP expression also suppresses the visually driven optokinetic response ( $n=34$ wild type and $n=45$ TeNT-Lc:EGFP larvae), locomotor activity ( $n=14$ wild-type and $n=50$ TeNT-Lc:EGFP larvae), and stimulusevoked calcium transients in tectal cells ( $n=8$ wild-type and $n=11$ TeNT-Lc:EGFP larvae). C, Optic tectum labeled with Oregon Green 488 BAPTA-1 AM. Tectal cell bodies are in the bottom right corner. Representative traces of calcium responses from wild-type (top) and TeNT-Lc: EGFP-expressing (bottom) larvae. Responses from individual cells are shown in gray, and the mean response is shown in black. $\boldsymbol{D}$. Summary of calcium imaging data. Each data point shows the mean response from each larvae. The horizontal line indicates the median for each group.

stream sequence of the zebrafish atoh7 gene (formerly ath5; Zebrafish Information Network) (Masai et al., 2003) was used to drive expression of GAL4, and blastomere injection of atoh7: GAL4 and UAS:TeNT-Lc:EGFP plasmids resulted in mosaic expression in RGCs. We found that when multiple, overlapping RGC axons were labeled in a single tectal hemisphere, it was difficult to reliably trace single arbors. Therefore, to ensure accurate morphometric measurement of axonal arbors, only larvae with expression in single or in nonoverlapping RGC axons were used for analysis. The TeNT-Lc:EGFP fusion protein served as an excellent marker of axonal arbor morphology, and in many cells expression persisted until $7 \mathrm{dpf}$, allowing the entire period of initial arbor development to be followed (Meyer and Smith,
2006). Figure $2 A$ shows expression of single RGC axons imaged over multiple days as they arborized in the tectum. RGC axons first reach the tectum between 2 and $3 \mathrm{dpf}$, and axons expressing EGFP, TeNT-Lc:EGFP, and TeNT-Lc mut:EGFP all showed a rapid increase in total arbor length (summed axon branch lengths) between 3 and $5 \mathrm{dpf}$, after which EGFP and TeNT-Lc mut:EGFP axon arbor length remains relatively constant (Fig. $3 A, B)$. There was no significant difference in the growth of axons expressing EGFP and TeNT-Lc mut:EGFP (repeated-measures ANOVA, Bonferroni's post hoc test, $p>0.05$ ). In contrast, RGC axons silenced by TeNT-Lc:EGFP fail to arrest growth at $5 \mathrm{dpf}$, resulting in RGCs with significantly longer arbors by $7 \mathrm{dpf}(130 \%$ of controls; repeated-measures ANOVA, $p<0.0001$, Bonferroni's post hoc test). The increase in arbor length could arise from an increase in axon arbor complexity (the number of branches per arbor), an increase in branch length, or through a combination of both processes. We found no significant difference in arbor complexity in cells expressing TeNT-Lc:EGFP compared with control axons (one-way ANOVA, Bonferroni's post hoc test, $p>0.05$ ) (Fig. $3 D)$. However, total branch length is increased in silenced axons, resulting in a $49 \%$ increase in axon arbor area in the tectum compared with control axons (one-way ANOVA, Bonferroni's post hoc test, $p<0.0001$ ) (Fig. 3C). These results show that synaptic activity is not required for axon branch formation but that synaptic activity is required for arresting the growth of RGC axonal arbors and for focusing them in the tectum.

Synaptic activity suppresses formation of transient filopodia Between 3 and $5 \mathrm{dpf}$, growth of RGC axon arbors in zebrafish is known to be a highly dynamic process characterized by the formation of numerous transient and highly dynamic filopodia, only a small fraction of which develop into stable branches in the mature arbor. Length of axonal branches is also dynamically regulated with changes occurring over just a few minutes (Kaethner and Stuermer, 1992; O'Rourke et al., 1994; Rajan et al., 1999; Meyer and Smith, 2006; Ruthazer et al., 2006). By 7 dpf, there are far fewer transient filopodia, and the established branches show little change in length over a period of many hours (Meyer and Smith, 2006). To explore the specific effects of suppressing synaptic activity on arbor growth dynamics, we performed timelapse imaging of single axonal arbors expressing TeNT-Lc:EGFP at $10 \mathrm{~min}$ intervals for a $10 \mathrm{~h}$ period (Fig. $4 \mathrm{~A}$ ) (supplemental Movie 2, available at www.jneurosci.org as supplemental material). Because the effects of single-cell silencing become apparent after $5 \mathrm{dpf}$, we performed time-lapse imaging between 5 and 7 dpf. As a control, we also imaged single axons expressing TeNT-Lc mut:EGFP between 5 and $7 \mathrm{dpf}$ (supplemental Movie 3, available at www.jneurosci.org as supplemental material). Figure $4 D$ shows that $94 \%$ of branches in TeNT-Lc mut:EGFPexpressing axons and $88 \%$ of branches in TeNT-Lc:EGFP axons that are present in the first frame of the time-lapse sequence persist until the final frame (the difference was not statistically significant). This demonstrates that synaptic activity is not required for branch maintenance, at least over the $10 \mathrm{~h}$ imaging period. Consistent with the increase in arbor length observed between 5 and $7 \mathrm{dpf}$ (Fig. 3B), we find that some branches in TeNT-Lc:EGFP-expressing axons show a significant increase in length during time-lapse imaging (Fig. $4 A, B$ ). This increase in individual branch lengths in TeNT-Lc:EGFP-expressing axons contributes to significant net arbor growth $(38.17 \pm 4 \mu \mathrm{m})$ during the $10 \mathrm{~h}$ imaging period compared with control axons $(-1 \pm$ $4 \mu \mathrm{m}$ ) (nonparametric one-way ANOVA, Dunn's post hoc test, $p<0.01$ ) (Fig. 4A,B) (supplemental Movies 2, 3, available at 
www.jneurosci.org as supplemental material). Surprisingly, time-lapse imaging also reveals that, although the majority of branches observed at the start of a timelapse sequence are stable, silenced axons form, on average, nearly three times as many new but transient processes as control axons (one-way ANOVA, Dunn's post hoc test, $p<0.05$ ) (Fig. $4 A, C$ ). These transient processes tended to be highly dynamic and of smaller diameter than the stable branches [most could be digitally excluded by applying a median filter with a radius of 3 pixels $(\sim 0.69 \mu \mathrm{m})]$. We therefore classified these transient processes as filopodia. The distribution of filopodia lifetimes (Fig. 4E) shows that the increase in total number of new filopodia formed by silenced axons is principally attributable to the formation of more of the short-lived (20 min bin) filopodia than control axons (two-way ANOVA, $p<0.0001)$. In addition to the short-lived filopodia, both TeNT-Lc:EGFP and control axons have a small population of filopodia with longer lifetimes (Fig. $4 A, E)$. We selected a threshold of $3 \mathrm{~h}$ to classify filopodia into categories of stable or transient. It has been shown previously that filopodia that persist often develop into stable axonal branches (Meyer and Smith, 2006). Interestingly, we find that, although silent axons show a dramatic increase in transient filopodia, the number of stable filopodia formed by silenced cells is not significantly different from active axons (two-way ANOVA, $p>0.05$ ). This is not too surprising, because we found no change in arbor complexity as a result of suppressing synaptic transmission, as shown in Figure 3D. Because we did not observe net increases in arbor length of control axons between 5 and $7 \mathrm{dpf}$, the increase in arbor length resulting from the formation of a small number of more sta-

ble filopodia by these axons is presumably balanced by branch elimination or branch retraction.

As mentioned above, the production of many highly dynamic transient filopodia is a feature of immature RGC axons (O’Rourke et al., 1994; Rajan et al., 1999; Meyer and Smith, 2006; Ruthazer et al., 2006; Campbell et al., 2007). Our time-lapse data showing that silenced axons continue to produce many transient filopodia suggest that presynaptic activity is required for arresting filopodia formation events and for arbor maturation. In support of this, we find no significant difference in the growth dynamics of silenced axons between 5 and $7 \mathrm{dpf}$ and those of immature, control axons expressing EGFP time-lapsed between 3 and $5 \mathrm{dpf}$ (one-way ANOVA, Dunn's post hoc test, $p>0.05$ for change in total branch length, new branches, and new filopodia lifetime) (Fig. $4 B, C, E$ ). Time-lapse imaging therefore reveals that presynaptic activity in RGC axons is not required for stabilizing axonal branches but that it is required for the transition from a highly dynamic arbor to a more stable one.

A
$5 \mathrm{dpf}$
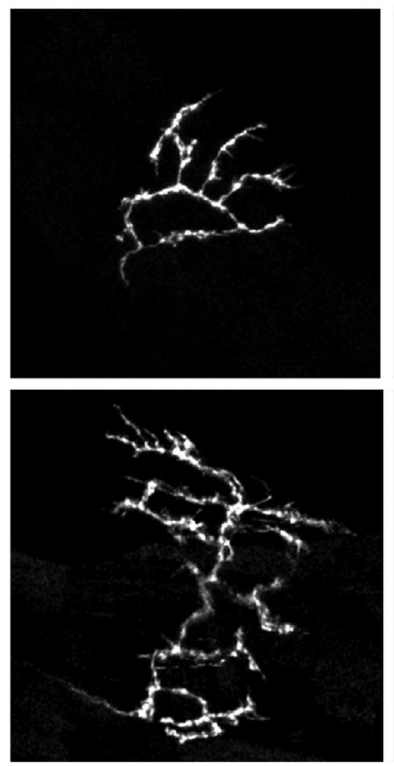

C

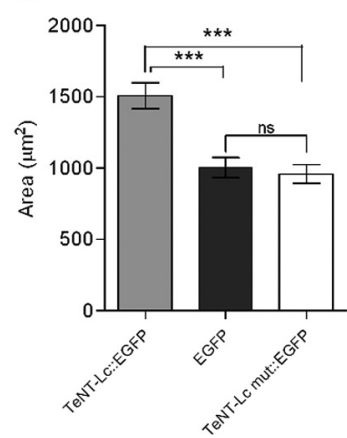

$7 \mathrm{dpf}$
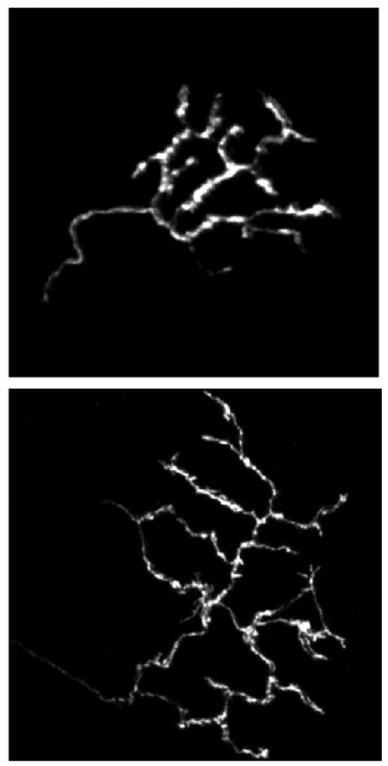

D

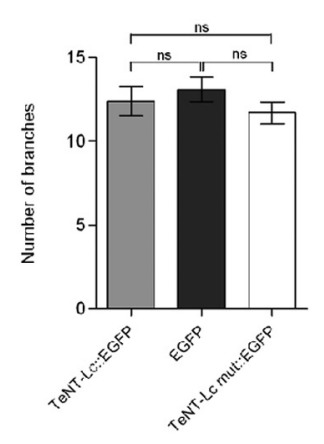

Figure 3. Single RGCs silenced by TeNT-LC:EGFP fail to arrest growth. $\boldsymbol{A}$, Single RGC axon expressing EGFP (top row) or TeNTLc:EGFP (bottom row) imaged at 3, 5, and $7 \mathrm{dpf}$ in the optic tectum of live zebrafish larvae. Scale bar, $30 \mu \mathrm{m}$. $\boldsymbol{B}$, Quantification of

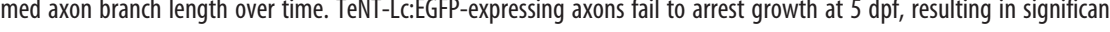
activity with TeNT-Lc:EGFP also results in increased axonal arbor coverage area in the tectum relative to controls. D, Suppressing presynaptic activity does not significantly alter axon branch number. All graphs show mean values \pm SEM from 21 EGFP-, 18 TeNT-Lc:EGFP-, and 13 TeNT-Lc mut:EGFP-expressing axons. For statistical analysis of total branch length over time, a repeatedmeasures ANOVA with Bonferroni's post hoc test was performed. For arbor area and branch number, statistical analysis was performed using a parametric one-way ANOVA with Bonferroni's post hoc test (ns, not significant; $p>0.05) .{ }^{* * *} p<0.001$.

\section{Activity-dependent competition regulates arbor size}

Activity-dependent competition between axons converging on common targets has been shown to modify patterns of synaptic connectivity in the visual cortex (Wiesel, 1982), olfactory system (Yu et al., 2004), neuromuscular junction (Buffelli et al., 2003), and the retinotectal projection of fish and frogs (Ruthazer et al., 2003; Hua et al., 2005). We therefore asked whether any of the features of TeNT-Lc:EGFP-expressing axons we had observed so far were a consequence of silencing just a single cell in a field of active ones. To test for an activity-based competition rule, we measured the growth of silenced axons when the activity of surrounding RGCs was also suppressed. To achieve this, we generated a transgenic line of zebrafish in which the regulatory regions of the zebrafish isl $2 b$ gene (formerly isl3; Zebrafish Information Network) were used to drive expression of GAL4. isl $2 b$ is expressed in all or nearly all RGCs (A. J. Pittman and C.-B. Chien, unpublished data). Injection of a plasmid bearing a UAS:TeNTLc:EGFP expression cassette flanked by Tol2 sites, along with 
A
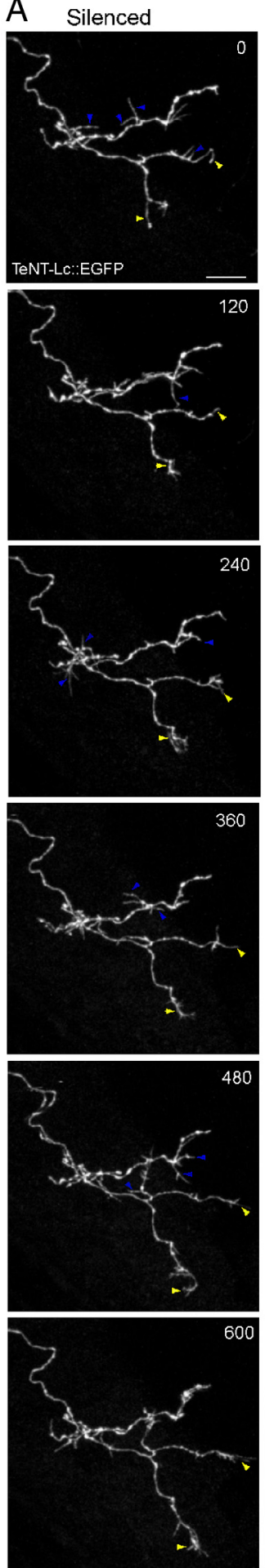

Key:

Growing branches

\section{- Transient filopodia}
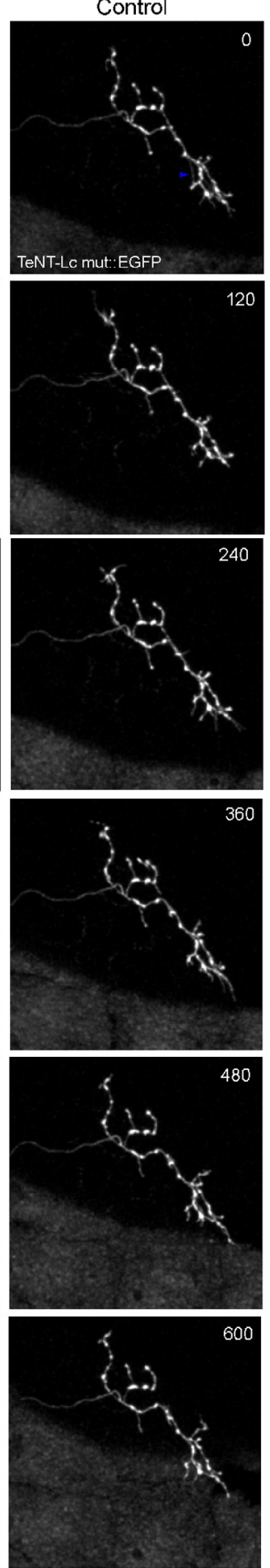

B
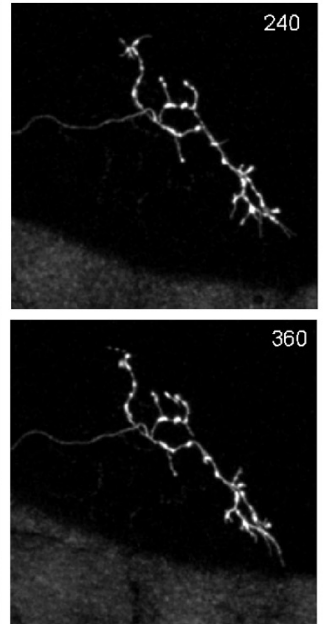

\section{E}

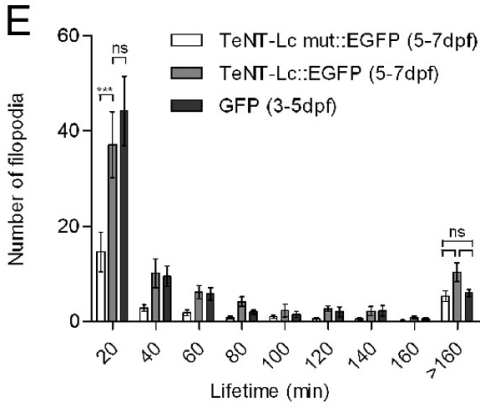

Figure 4. Time-lapse analysis of RGC axon arbor dynamics. $A$, Series of still images taken from $10 \mathrm{~h}$ time-lapse movies between 6 and $7 \mathrm{dpf}$, at imaging interval of $10 \mathrm{~min}$. Axons silenced by TeNT-Lc:EGFP expression (left column) show significant net growth of some axonal branches (yellow arrowheads) and numerous transient branches (blue arrowheads). Axons expressing TeNT-Lc mut:EGFP (right column) show little or no net growth over the imaging period and far fewer transient branches. Time in minutes is indicated in the top right corner. Scale bar, $30 \mu \mathrm{m}$. $\boldsymbol{B}$, Quantification of net change in total axon arbor length over the $10 \mathrm{~h}$ imaging period. Silenced axons grow significantly more than control TeNT-Lc mut:EGFP axons. C, Number of new branch formation events during a $10 \mathrm{~h}$ time lapse. Silenced axons form significantly more new branches than controls. $\boldsymbol{D}$, Number of branches that
mRNA encoding Tol2 transposase into isl2b:GAL4 transgenic embryos (hereafter referred to as TeNT-Lc:EGFP Tol2), resulted in widespread expression in the retinal ganglion cell layer of the retina (Fig. $5 A$ ). This widespread expression is consistent with previous observations showing that the Tol2 transposon is highly active in somatic tissues (Balciunas et al., 2006). Although expression levels were generally low, we could also clearly see dense and extensive labeling of RGC axons in the tectal neuropil (Fig. 5B). Occasionally, we observed isolated axons, strongly labeled by TeNT-Lc:EGFP, against the background of more weakly labeled axons. This is presumably attributable to high expression levels derived from the mosaic inheritance of episomal TeNT-Lc:EGFP Tol2 plasmid (Fig. 5B). To visualize the morphology of single RGC axons in TeNT-Lc:EGFP Tol2 fish (in instances in which single axons were not brightly labeled with TeNT-Lc:EGFP), we coinjected a plasmid encoding the red fluorescent protein Tandem-dimer Tomato (UAS:TdT) (Shaner et al., 2004) (Fig. $5 A, B)$. As Figure $5 B$ illustrates, it was not uncommon to see that the axons that were brightly labeled with TeNT-Lc:EGFP were also brightly labeled with TdT. This is presumably attributable to the mosaic inheritance of both TdT and TeNT-Lc:EGFP Tol2 plasmids in a single RGC. Under conditions of widespread suppression of synaptic activity in RGCs, single axon arbor length, arbor area, and branch number were not statistically different from EGFP-labeled control axons in wild-type larvae (Fig. 5C-E). However, total arbor length and arbor area were both significantly reduced compared with single arbors that expressed TeNT-Lc:EGFP. Although expression of TeNT-Lc:EGFP

were present in the first frame of a time lapse that persisted until the final frame, $10 \mathrm{~h}$ later. Branch stability is unaffected by TeNT-Lc:EGFP expression. $\boldsymbol{E}$, Histogram of branch lifetime. Silenced axons produce many more of the shortest-lived branches, but the number of longer-lived branches is unaffected by suppressing synaptic function. $\boldsymbol{B}, \boldsymbol{C}$, and $\boldsymbol{E}$ also demonstrate that there is no significant difference in the amount of net growth or new branches formed by TeNT-Lc:EGFPexpressing axons during a $10 \mathrm{~h}$ period between 5 and $7 \mathrm{dpf}$ and immature EGFP axons imaged for $10 \mathrm{~h}$ between 3 and $5 \mathrm{dpf}$ $(n=4)$. For statistical analysis of branch lifetime, a two-way ANOVA with Bonferroni's post hoc test was performed $\left({ }^{* * *} p<0.0001\right)$. For all other analyses, a nonparametric oneway ANOVA, followed by a Dunn's post hoc test was used (ns, not significant; $p>0.05) .{ }^{*} p<0.05 ;{ }^{* *} p<0.01$. All graphs show mean \pm SEM values of four EGFP-labeled axons, six TeNT-Lc:EGFP-expressing axons, and six axons expressing TeNT-Lc mut:EGFP. 
using the Tol2 system appeared widespread in RGCs, it might have been the case that not all RGCs expressed TeNTLc:EGFP. As a second approach to test for a competition-based rule, we injected mRNA encoding TeNT-Lc:EGFP into single-cell stage zebrafish embryos. This resulted in global suppression of synaptic activity, assayed by paralysis of injected larvae. In agreement with a previous study, we find that the gross anatomy of the retina and tectum is unaffected by global suppression of neural activity in zebrafish (supplemental Fig. 2, available at www.jneurosci.org as supplemental material) (Nevin et al., 2008). Using the mosaic labeling strategy outlined above, a single RGC axon was labeled with TeNT-Lc:EGFP in TeNT-Lc:EGFP mRNAinjected larvae. Because of the high levels of expression achieved using the GAL4-UAS system, EGFP expression in a single mosaically labeled cell was clearly visible against the diffuse EGFP expression resulting from mRNA injection (Fig. 6A) (supplemental Movie 4, available at www.jneurosci.org as supplemental material). Similar to the results obtained using the Tol2 system, we find that single arbors grown under conditions of global synaptic silencing are morphologically indistinguishable from wild-type axons (Fig. 5C-E); again, their area and total length were significantly reduced compared with isolated silenced arbors. This "rescue" from the effects of suppressing activity in a single cell indicates that competition from neighboring axons, eliminated by suppression of activity in neighboring axons, may be responsible for the overgrowth phenotype observed when only a single cell is silenced.

\section{Formation of transient filopodia is not} regulated by competing neighbors

Time-lapse imaging of a single silenced axon revealed two distinct effects of suppressing synaptic activity on arbor dynamics. First, there is a significant increase in arbor length and area, and, second, the formation of many transient filopodia that are characteristic of immature axons persist in singly silenced axons. To tease apart specific aspects of arbor growth dy-

namics that are regulated by competitive mechanisms, we performed time-lapse imaging of singly labeled RGC axon for a period of $10 \mathrm{~h}$ between 5 and $7 \mathrm{dpf}$ in a globally silenced (TeNTLc:EGFP mRNA-injected) background (Fig. 6A) (supplemental Movie 4, available at www.jneurosci.org as supplemental material). Consistent with our observations that single arbors in globally silenced larvae are of a normal size at $7 \mathrm{dpf}$, we found little change in total arbor length during the $10 \mathrm{~h}$ imaging period $(2.2 \pm 3.2 \mu \mathrm{m})$ (Fig. 6B). Surprisingly, however, we find that the formation of many transient filopodia that we observed when silencing a single axon is also evident when activity is suppressed

C ${ }^{* * *} p<0.001$
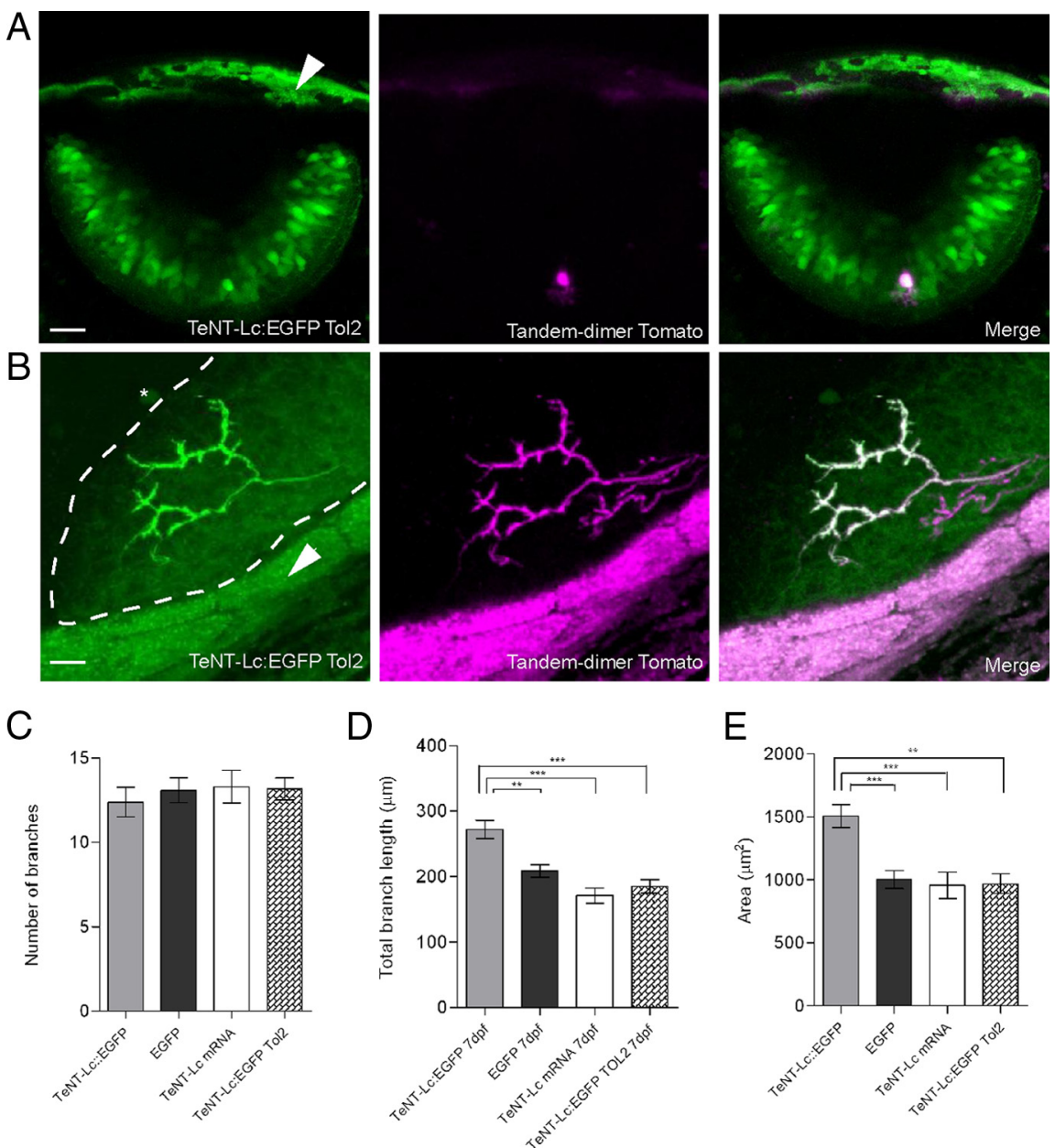

Figure 5. Axonal arbor morphology is normal when activity is blocked in all RGCs. $\boldsymbol{A}$, Injection of transposase mRNA and a plasmid encoding TeNT-Lc:EGFP flanked by To/2 sites into the $/ s / 2 b$ :GAL4 transgenic line of zebrafish results in widespread expression of TeNT-Lc:EGFP specifically in RGCs (left). Coinjection of a plasmid encoding TdT (without Tol2 sites) results in mosaic labeling of isolated RGCs (middle). The red is displayed as magenta. The red and the green channels are merged in the far right panel. Autofluorescence from the skin is indicated by the arrowhead in the left panel. $\boldsymbol{B}$, Single axon arbor morphology visualized in the ectum of TeNT-Lc:EGFP Tol2 fish. EGFP fluorescence from widespread expression of TeNT-Lc:EGFP in RGC axons is displayed in the t panel. The limit of the tectal neuropil is marked with a dashed line. Sparse labeling in tectal cells marked with * is occasionally observed. Skin autofluorescence is marked with an arrowhead. In the example shown, a single axon is also brightly labeled with TeNT-Lc:EGFP against a background of less strongly labeled axons. A single axon labeled with TdT, displayed as magenta, is synaptic activity in a single cell or globally. D, Quantification of summed axon branch lengths at $7 \mathrm{dpf}$. Axons grown under conditions of widespread of synaptic transmission (TeNT-Lc:EGFP Tol2 and TeNT-Lc mRNA) are not statistically different from shown. $\boldsymbol{E}$, Arbor area is also reduced to control levels when synaptic activity is suppressed globally. All graphs show mean \pm SEM 作 21 EGFP-labeled axons in nonsilenced background, 18 single silenced axons (TeNT-Lc:EGFP), 13 axons in TeNT-LC mRNAwo-way ANOVA with Bonferroni's post hoc test was performed. For arbor area and branch number, statistical analysis was performed using a parametric one-way ANOVA with Bonferroni's post hoc test (ns, not significant; $p>0.05$ ). ${ }^{* *} p<0.01$;

globally, i.e., axons imaged in a globally silenced background produce as many new, transient filopodia $(92 \pm 11)$ as a single silent axon $(98 \pm 18)$ (one-way ANOVA, $p>0.05)$ (Fig. $6 A, C$ ) (supplemental Movie 4, available at www.jneurosci.org as supplemental material). Moreover, the distribution of filopodia lifetimes is not significantly different when presynaptic function is suppressed in a single cell or globally (Fig. 6D). These results suggest that filopodia formation events and branch extension are regulated independently of one another: branch extension (and hence arbor territory) is regulated by activity in "competing" neighbors, whereas filopodia formation is reg- 


\section{A}
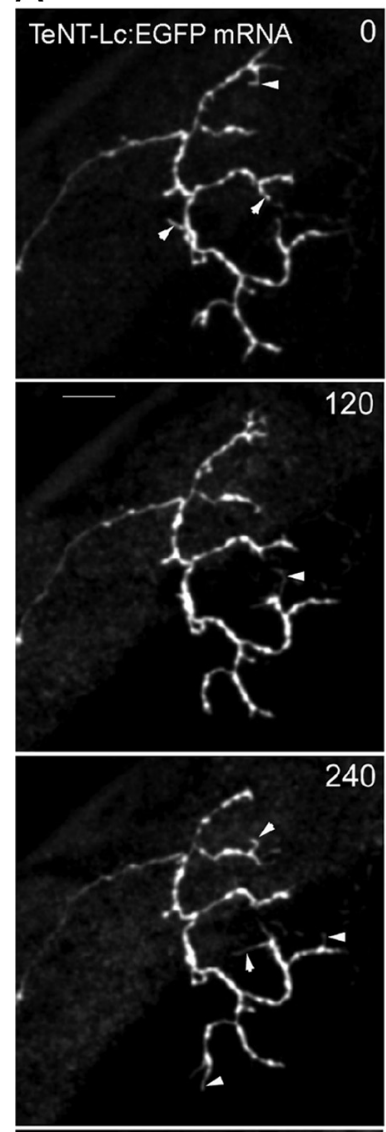

360
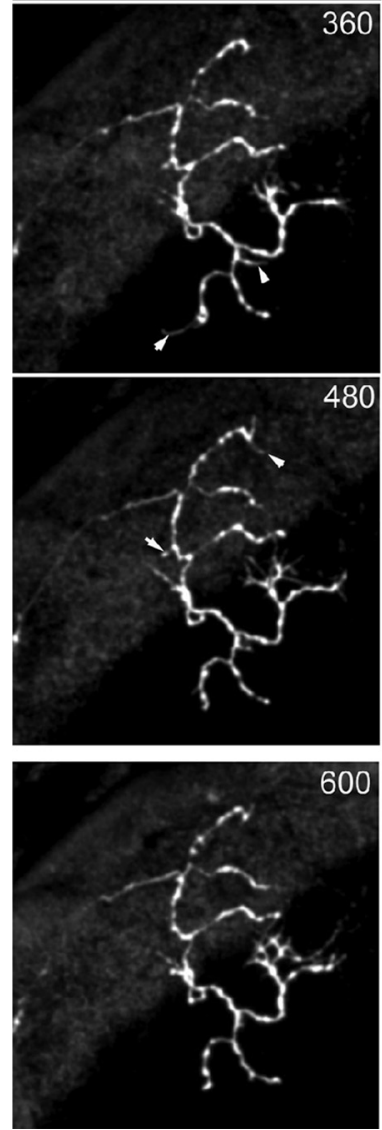

600

Figure 6. Time-lapse imaging of single axonal arbors under conditions of global suppression of synaptic activity. $\boldsymbol{A}$, Series of still images taken from $10 \mathrm{~h}$ time-lapse movies between 6 and 7 ulated by synaptic activity, regardless of activity in neighboring axons.

\section{Competition for arbor territory is NMDAR dependent}

Implicit in the observation that NMDAR antagonists decrease the retinotopic precision of RGC innervation is the existence of an activity-dependent retrograde signal, generated postsynaptically, that alters the behavior of RGC axons (Cline and ConstantinePaton, 1989; Rajan et al., 1999; Ruthazer et al., 2003). To investigate the involvement of NMDAR signaling in activity-based competition between RGC axons, we silenced a single RGC with TeNT-Lc:EGFP and raised larvae between 3 and $7 \mathrm{dpf}$ in rearing medium containing the NMDAR antagonist MK-801. Exposure to $\mathrm{MK}-801$ in the rearing medium has been demonstrated to block all NMDAR-mediated visual responses in tectal neurons (O'Rourke et al., 1994; Ruthazer et al., 2003). Consistent with previous observations, we find that treatment of control axons with MK-801 did not significantly alter total branch length, arbor area, or branch number (two-way ANOVA, $p>0.05$ ) (Fig. 7A-D) (O'Rourke et al., 1994; Rajan et al., 1999). However, with MK-801 treatment, the morphological development of single TeNT-Lc:EGFP-expressing axons was now indistinguishable from control axons (for all measured parameters: two-way ANOVA, $p>0.05$ ) (Fig. 7A-D). This pharmacological rescue from the growth-promoting effects of blocking presynaptic function in a single RGC suggests that NMDAR and retrograde signaling may be involved in the competitive form of plasticity that we have observed.

\section{Suppressing synaptic activity in all RGCs disrupts the convergence of axons in the tectum}

Although suppression of synaptic activity in all RGCs does not alter single axon arbor morphology, the convergence or relationship of one axonal arbor relative to another may be disrupted. To address whether global suppression of synaptic activity also disrupts retinotopy, we performed small focal injections of the lipophilic neuronal tracer DiI into a nasodorsal patch of retina in 6 dpf TeNT-Lc:EGFP Tol2 larvae or larvae injected with TeNT-Lc: EGFP mRNA. Anterograde transport of DiI from the retina allowed the projection of axons to the tectum to be analyzed. The projection of RGC axons onto the tectum of both TeNT-Lc:EGFP Tol2 fish and TeNT-Lc:EGFP mRNA-injected fish is topographic but is less well defined than in wild type (Fig. $8 \mathrm{~A}$ ). In wild-type larvae, axons form a tightly focused termination zone characterized by a central region of increased fluorescence staining intensity (illustrated by applying a thermal lookup table to the

$\mathrm{dpf}$, with imaging interval of $10 \mathrm{~min}$. Axons grown under conditions of global suppression of synaptic activity show numerous transient branches (white arrowheads) but little net growth over the $10 \mathrm{~h}$ imaging period. $\boldsymbol{B}$, Quantification of net arbor length change over the $10 \mathrm{~h}$ imaging period. Similar to control axons imaged in nonsilent backgrounds between 5 and $7 \mathrm{dpf}$ (TeNT-Lc mut:EGFP at 5-7 dpf), axons grown under conditions of global suppression of synaptic activity (TeNT-Lc mRNA at 5-7dpf) show very little net growth. For comparison, data for singly silenced axons between 5 and $7 \mathrm{dpf}$ (TeNT-Lc:EGFP at 5-7 dpf) are also shown. C, Cells grown under conditions of global silencing produce as many new transient branches as single silenced axons. D, Histogram showing that the distribution of new branch lifetimes is the same under conditions of single-cell and global suppression of synaptic activity. For comparison, values for axons expressing TeNT-LC mut:EGFP between 5 and $7 \mathrm{dpf}$ are also shown. For statistical analysis of branch lifetime, a two-way ANOVA with Bonferroni's post hoc test was performed $\left({ }^{* * *} p<\right.$ $0.0001)$. All other tests were performed using a nonparametric one-way ANOVA, followed by a Dunn's post hoc test (ns, not significant; $p>0.05$ ). ${ }^{*} p<0.05$. All graphs show mean \pm SEM values of five axons imaged under conditions of global silencing (TeNT-Lc mRNA at 5-7 dpf), six single silenced axons (TeNT-Lc:EGFP at 5-7 dpf), and six control axons under nonsilenced conditions (TeNT-Lc mut:EGFP at 5-7 dpf). 
fluorescence image). Axons in larvae silenced by TeNT-Lc had a more diffuse termination zone that either lacked regions of more intense labeling altogether or had smaller, fragmented regions of brighter fluorescence labeling (Fig. 8A). We also assessed the refinement of the retinotectal map from the degree of scatter of RGC axons labeled by injection of DiI into the retina. By plotting the tectal projection area as a function of area labeled in the retina, we found that wild-type larvae tended to show a positive correlation between these two measures. However, in TeNT-Lc:EGFP Tol2 larvae and in larvae injected with TeNT-Lc:EGFP mRNA, the best-fit lines through these datasets are nearly horizontal. This suggests that tectal projection area is essentially independent of retinal injection area, i.e., the projection of RGC axons is more random than in wild-type larvae (Fig. 8B). These data indicate that, although single arbor morphology appears normal in synaptically silenced zebrafish, the convergence of RGC axons in the tectum is perturbed.

\section{Discussion}

To provide a detailed description of the influence of presynaptic function on the growth of axonal arbors, we imaged RGC axons expressing a fusion of TeNT-Lc to EGFP at high temporal and spatial resolution as they arborized in the optic tectum of zebrafish larvae. TeNT-Lc has been used widely to look at the function of neural circuits (White et al., 2001), and the role of synaptic activity in regulating circuit assembly (Yu et al., 2004; Kerschensteiner et al., 2009). We have several lines of evidence showing that, when fused to fluorescent proteins, TeNT-Lc retains its proteolytic activity and suppresses synaptic transmission. First, using the genetically encoded reporter of presynaptic activity sypHy, we found that evoked release is completely abolished in dissociated hippocampal neurons overexpressing TeNT-Lc:TdT and spontaneous release is dramatically reduced. Similarly, using FM dyes, we found that neurons expressing TeNT-Lc:EGFP showed a marked reduction in vesicle cycling at presynaptic terminals. Finally, we show that ubiquitous expression of TeNT-Lc: EGFP by injection of mRNA into zebrafish embryos suppresses locomotor activity, visual background adaptation, the optokinetic response, and stimulus-evoked calcium transients in tectal cells. mRNA injected into zebrafish embryos is typically shortlived (typically 1-2 d). The prolonged paralysis of larvae (until at least $7 \mathrm{dpf}$ in many cases) suggests that TeNT-Lc:EGFP protein is very stable, very potent, or both. It is therefore highly likely that, with the high levels of expression achieved using the GAL4-UAS system, synaptic transmission is blocked in single RGCs expressing TeNT-Lc:EGFP over the entire period that axons were imaged.

We find that presynaptic activity is not required for generating an arbor with an appropriate number of stable branches. Thus,
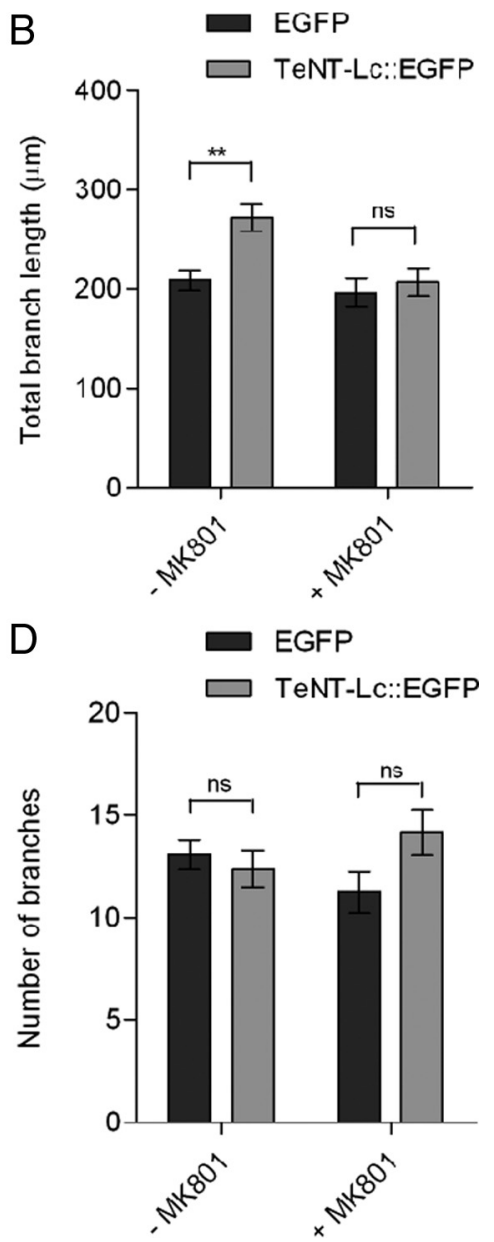

Figure 7. Morphology of single cells silenced by TeNT-Lc:EGFP is rescued by bath application of the NMDAR antagonist MK-801. , B, Treatment with MK-801 did not significantly alter the growth of control (EGFP)-expressing axons ( $p>0.05)$, but MK-801 reatment with MK-801 also reverses the increase in arbor area induced by single-cell expression of TeNT-Lc:EGFP. Note that the a of control axons is unaffected by MK-801 treatment. D, Branch number of control and silenced axons is unaffected by MK-80 single silenced axons (TeNT-Lc:EGFP) minus MK-801, and 15 TeNT-Lc:EGFP-expressing axons plus MK-801. For statistical analysis, a parametric tw0-way ANOVA with Bonferroni's post hoc test was performed (ns, not significant; $p>0.05) .{ }^{* *} p<0.01$.

although synaptic sites are correlated with branch stabilization, the synapse need not be functional to maintain axonal branches. However, presynaptic function does regulate other distinct aspects of axonal arbor development. First, silent axons continue to produce many transient filopodia, a feature of immature axons. This suggests that presynaptic activity is required for arbor maturation. A previous time-lapse study in zebrafish has shown that RGC filopodia formation occurs almost exclusively at newly formed synaptic sites and that filopodia formation is suppressed at synapses more than a few hours old (Meyer and Smith, 2006). Our data suggest that synaptic activity is required for this developmental transition at synapses to occur. In hippocampal neurons, glutamate has been shown to suppress filopodial motility, suggesting that release of neurotransmitter, abolished by TeNT-Lc expression, may act in an autocrine manner to suppress filopodia formation (Chang and De Camilli, 2001). Despite the fact that silenced axons produce many more dynamic filopodia than control axons, these filopodia tend to be short-lived (generally $<20 \mathrm{~min}$ ). However, imaging of immature RGC axons expressing a fluorescent presynaptic marker protein has shown that even very transient filopodia are capable of making nascent syn- 
A
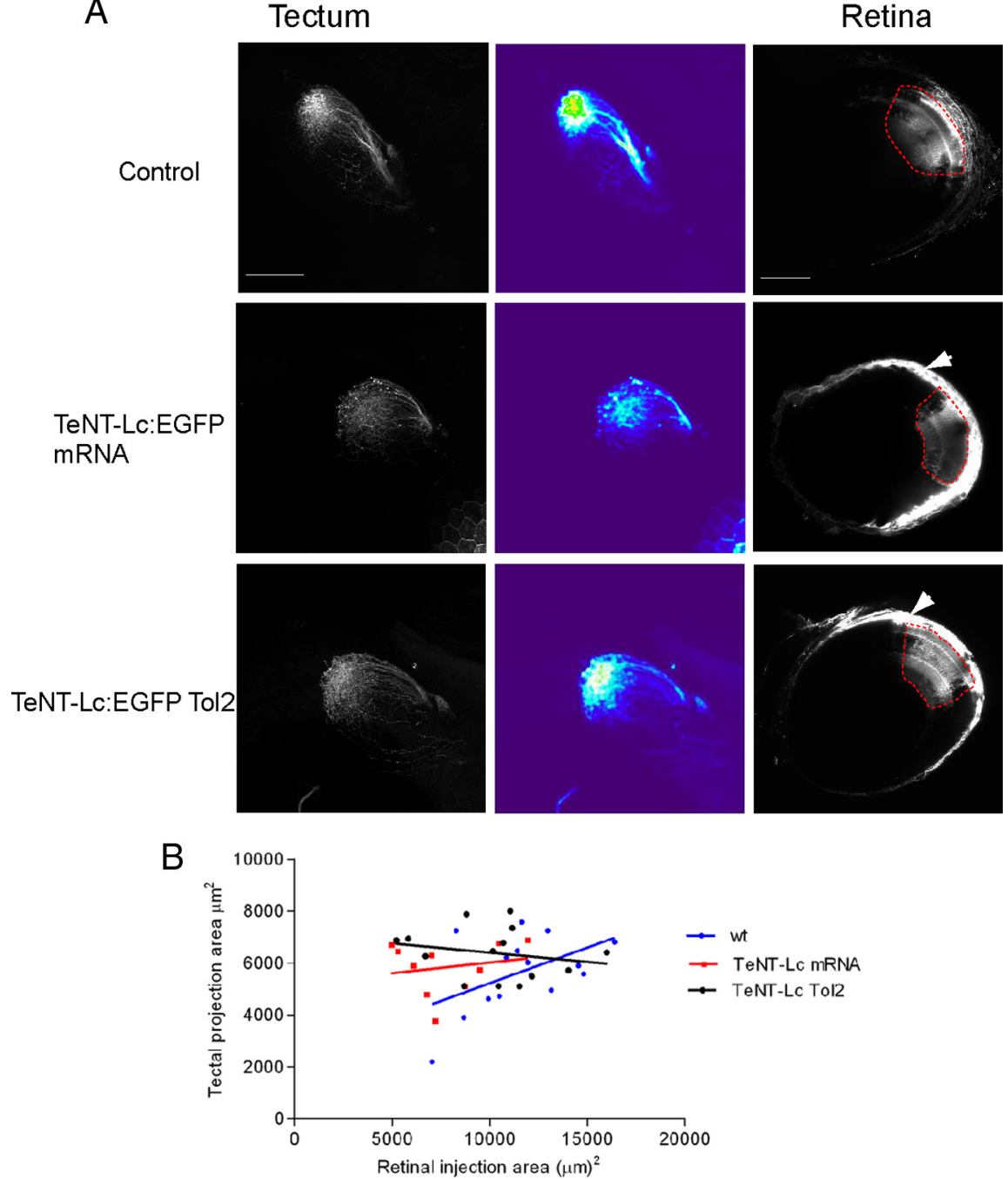

Figure 8. Global suppression of synaptic activity disrupts convergence of RGC axons in the tectum. $A$, Dil labeling of the retinotectal projection of nasodorsal RGCs in wild-type (control, top row), TeNT-Lc:EGFP mRNA-injected (middle row), and TeNTLc:EGFP Tol2 (bottom row) zebrafish larvae at $6 \mathrm{dpf}$. Dorsal views of labeled RGCs in the tectum are shown in the right images, and the corresponding injection site in the retina is shown in the far left images. The area measured in the retina is indicated by the dashed line. Areas of fluorescence outside this area correspond to Dil labeling of the skin (arrowheads). To better illustrate variations in fluorescence labeling intensity, a thermal lookup table was applied to images of the RGC projection fields (middle images). Note the more dispersed projection field and absence of a central bright region of fluorescence in the silenced larvae. Scale bars, $50 \mu \mathrm{m}$. B, Scatter plot of tectal projection area as a function of retinal injection area. Wt, Wild type.

aptic contacts (Alsina et al., 2001; Meyer and Smith, 2006; Ruthazer et al., 2006). Therefore, in the normal retinotectal projection, activity-dependent cessation of filopodia formation would be important for maturation of the retinotopic map and for enabling stable visual system function. Time-lapse imaging reveals that the failure to arrest formation of filopodia is also seen when synaptic function is suppressed in all RGC axons. Thus, in contrast to arbor territory (see below), filopodia formation is regulated by presynaptic function regardless of activity in neighboring axons.

A second consequence of suppressing presynaptic function is that singly silenced axons fail to arrest growth of well established branches. This results in significant expansion of territory occupied by the axonal arbor in the tectum. However, when neighboring RGCs are also silenced, single arbor area is restored to normal levels. This finding suggests that silent axons are capable of maintaining an appropriately sized territory within the tectum but are prevented from doing so by more active neighbors. Thus, the ability of axons to arrest growth and stably occupy territory is an activity-dependent, "competitive" process. Our results are remarkably similar to those seen in olfactory sensory neurons silenced by TeNT-Lc (Yu et al., 2004). In this study, suppression of synaptic release in all olfactory sensory neurons by TeNT-Lc expression did not alter the precise targeting and convergence of axons onto olfactory bulb glomeruli. However, severe phenotypes were observed when TeNT-Lc was expressed in small populations of neurons expressing a given odorant receptor. Under these competitive conditions, silenced axons failed to converge on the appropriate glomeruli and ultimately strayed over the entire medial surface of the bulb. A plausible scenario to explain these observations is that axons converging on common targets compete with one another based on relative activity levels for a "stop growing" signal, thereby allowing them to stably occupy an appropriate territory within the target area. The cellular mechanisms underlying the competition we describe are unknown, but the observation that the NMDAR antagonist MK-801 also reverses the effects of single-cell silencing implicates the postsynaptic cell, acting through a retrograde signal, in the competitive process. Many candidate retrograde messengers have been proposed, including neurotrophins, cell adhesion molecules, and nitric oxide (Regehr et al., 2009). Because MK-801 was added to the rearing medium, retinal function is likely to be perturbed, and this in turn could modify the development of RGC axonal arbors. Bath application of MK- 801 could also modify axon arbor growth directly by acting on presynaptic NMDARs. However, MK-801 only appears to modulate the growth of singly silenced RGC axons and does not alter the morphology of single control arbors expressing EGFP. Thus, MK-801 treatment does not induce obvious structural changes in axons unless they are grown under competitive conditions.

Although single arbor morphology appears normal under global suppression of synaptic activity, the convergence of RGC axons in the tectum appeared to be disrupted. Our data are therefore consistent with a previous study in zebrafish showing that widespread suppression of electrical activity in RGCs also disrupts the precise neighbor relationships that are the central feature of retinotopic maps (Gnuegge et al., 2001).

Our results are, however, diametrically opposed to those of a previous study that used very similar approaches to the ones we have used here (Hua et al., 2005). In the study by Hua et al. (2005), a mutant form of VAMP2/Synaptobrevin 2 was used to suppress presynaptic activity in single RGC axons (Sørensen et al., 2002). This manipulation resulted in smaller axonal arbors. In contrast, using TeNT-Lc, we find that single arbors are enlarged. What could account for these differences? An intriguing explanation is that the degree of presynaptic suppression achieved with 
the two approaches is different. Overexpression of the VAMP2 mutant has been shown to suppress stimulus-evoked vesicular release but does not abolish it entirely (Sørensen et al., 2002). Moreover, the nonfunctional VAMP2 mutant suppresses synaptic function by competing with endogenous VAMP2 for binding sites on other SNARE ( $N$-ethylmaleimide-sensitive factor attachment protein receptor) proteins. Thus, the efficacy of the manipulation will be highly sensitive to levels of expression of the VAMP2 mutant, something that is difficult to control. For these reasons, it seems likely that, in RGCs expressing the VAMP2 mutant, presynaptic function is suppressed but not abolished entirely. Conversely, we have shown that TeNT-Lc is highly effective at blocking both stimulus-evoked and spontaneous release of synaptic vesicles. The weak residual activity at presynaptic terminals of RGCs expressing the VAMP2 mutant could therefore induce plasticity mechanisms analogous to long-term depression (LTD), which in the developing retinotectal projection may lead to synapse elimination. Long-term potentiation and LTD have both been demonstrated to occur at retinotectal synapses (Zhang et al., 1998, 2000; Vislay-Meltzer et al., 2006). Because synapses appear to be correlated with both stabilization and formation of axonal branches (Alsina et al., 2001; Meyer and Smith, 2006; Ruthazer et al., 2006), synapse elimination would lead to both an increased rate of branch elimination and a reduced rate of branch formation. Both of these would result in smaller axonal arbors. LTD, by definition, only occurs at synapses with at least some activity. Therefore, in axons that express TeNT-Lc:EGFP, in which synaptic activity is almost abolished entirely, LTD and synapse elimination are not induced, permitting branches to extend beyond their normal territory. Thus, different levels of suppression of synaptic transmission could have very different consequences for the growth of axonal arbors. Compelling support for this idea comes from studies of LTD induced by monocular deprivation in the visual cortex. Eye-lid suture, which preserves spontaneous activity in the retina, causes significantly greater depression of deprived-eye responses in the cortex than does treatment with tetrodotoxin, which eliminates all activity (Rittenhouse et al., 1999).

Together, our results show that presynaptic function is not required for axon branch maintenance but that it is a cellautonomous requirement for arresting branch formation events. Moreover, we find a novel role for activity-dependent interaxonal competition in stopping growth of arbors and for defining arbor territory in the tectum. Both of these processes are crucial for development of a finely tuned retinotopic map and for stable visual system function.

\section{References}

Alsina B, Vu T, Cohen-Cory S (2001) Visualizing synapse formation in arborizing optic axons in vivo: dynamics and modulation by BDNF. Nat Neurosci 4:1093-1101.

Asakawa K, Suster ML, Mizusawa K, Nagayoshi S, Kotani T, Urasaki A, Kishimoto Y, Hibi M, Kawakami K (2008) Genetic dissection of neural circuits by Tol2 transposon-mediated Gal4 gene and enhancer trapping in zebrafish. Proc Natl Acad Sci U S A 105:1255-1260.

Balciunas D, Wangensteen KJ, Wilber A, Bell J, Geurts A, Sivasubbu S, Wang X, Hackett PB, Largaespada DA, McIvor RS, Ekker SC (2006) Harnessing a high cargo-capacity transposon for genetic applications in vertebrates. PLoS Genet 2:e169.

Brockerhoff SE (2006) Measuring the optokinetic response of zebrafish larvae. Nat Protoc 1:2448-2451.

Buffelli M, Burgess RW, Feng G, Lobe CG, Lichtman JW, Sanes JR (2003) Genetic evidence that relative synaptic efficacy biases the outcome of synaptic competition. Nature 424:430-434.
Campbell DS, Stringham SA, Timm A, Xiao T, Law MY, Baier H, Nonet ML, Chien CB (2007) Slitla inhibits retinal ganglion cell arborization and synaptogenesis via Robo2-dependent and -independent pathways. Neuron 55:231-245.

Chang S, De Camilli P (2001) Glutamate regulates actin-based motility in axonal filopodia. Nat Neurosci 4:787-793.

Cline HT, Constantine-Paton M (1989) NMDA receptor antagonists disrupt the retinotectal topographic map. Neuron 3:413-426.

Galli T, Chilcote T, Mundigl O, Binz T, Niemann H, De Camilli P (1994) Tetanus toxin-mediated cleavage of cellubrevin impairs exocytosis of transferrin receptor-containing vesicles in $\mathrm{CHO}$ cells. J Cell Biol 125:1015-1024.

Gnuegge L, Schmid S, Neuhauss SC (2001) Analysis of the activity-deprived zebrafish mutant macho reveals an essential requirement of neuronal activity for the development of a fine-grained visuotopic map. J Neurosci 21:3542-3548.

Granseth B, Odermatt B, Royle SJ, Lagnado L (2007) Clathrin-mediated endocytosis: the physiological mechanism of vesicle retrieval at hippocampal synapses. J Physiol 585:681-686.

Hua JY, Smear MC, Baier H, Smith SJ (2005) Regulation of axon growth in vivo by activity-based competition. Nature 434:1022-1026.

Huberman AD, Feller MB, Chapman B (2008) Mechanisms underlying development of visual maps and receptive fields. Annu Rev Neurosci 31:479-509.

Kaethner RJ, Stuermer CA (1992) Dynamics of terminal arbor formation and target approach of retinotectal axons in living zebrafish embryos: a time-lapse study of single axons. J Neurosci 12:3257-3271.

Kerschensteiner D, Morgan JL, Parker ED, Lewis RM, Wong RO (2009) Neurotransmission selectively regulates synapse formation in parallel circuits in vivo. Nature 460:1016-1020.

Köster RW, Fraser SE (2001) Tracing transgene expression in living zebrafish embryos. Dev Biol 233:329-346.

Kwan KM, Fujimoto E, Grabher C, Mangum BD, Hardy ME, Campbell DS, Parant JM, Yost HJ, Kanki JP, Chien CB (2007) The Tol2kit: a multisite gateway-based construction kit for Tol2 transposon transgenesis constructs. Dev Dyn 236:3088-3099.

Li Z, Burrone J, Tyler WJ, Hartman KN, Albeanu DF, Murthy VN (2005) Synaptic vesicle recycling studied in transgenic mice expressing synaptopHluorin. Proc Natl Acad Sci U S A 102:6131-6136.

Masai I, Lele Z, Yamaguchi M, Komori A, Nakata A, Nishiwaki Y, Wada H, Tanaka H, Nojima Y, Hammerschmidt M, Wilson SW, Okamoto H (2003) N-cadherin mediates retinal lamination, maintenance of forebrain compartments and patterning of retinal neurites. Development 130:2479-2494.

Meyer MP, Smith SJ (2006) Evidence from in vivo imaging that synaptogenesis guides the growth and branching of axonal arbors by two distinct mechanisms. J Neurosci 26:3604-3614.

Nevin LM, Taylor MR, Baier H (2008) Hardwiring of fine synaptic layers in the zebrafish visual pathway. Neural Dev 3:36.

Nicol X, Voyatzis S, Muzerelle A, Narboux-Nême N, Südhof TC, Miles R, Gaspar P (2007) cAMP oscillations and retinal activity are permissive for ephrin signaling during the establishment of the retinotopic map. Nat Neurosci 10:340-347.

Niell CM, Smith SJ (2005) Functional imaging reveals rapid development of visual response properties in the zebrafish tectum. Neuron 45:941-951.

Niell CM, Meyer MP, Smith SJ (2004) In vivo imaging of synapse formation on a growing dendritic arbor. Nat Neurosci 7:254-260.

O'Rourke NA, Cline HT, Fraser SE (1994) Rapid remodeling of retinal arbors in the tectum with and without blockade of synaptic transmission. Neuron 12:921-934.

Rajan I, Witte S, Cline HT (1999) NMDA receptor activity stabilizes presynaptic retinotectal axons and postsynaptic optic tectal cell dendrites in vivo. J Neurobiol 38:357-368.

Regehr WG, Carey MR, Best AR (2009) Activity-dependent regulation of synapses by retrograde messengers. Neuron 63:154-170.

Rittenhouse CD, Shouval HZ, Paradiso MA, Bear MF (1999) Monocular deprivation induces homosynaptic long-term depression in visual cortex. Nature 397:347-350.

Ruthazer ES, Cline HT (2004) Insights into activity-dependent map formation from the retinotectal system: a middle-of-the-brain perspective. J Neurobiol 59:134-146. 
Ruthazer ES, Akerman CJ, Cline HT (2003) Control of axon branch dynamics by correlated activity in vivo. Science 301:66-70.

Ruthazer ES, Li J, Cline HT (2006) Stabilization of axon branch dynamics by synaptic maturation. J Neurosci 26:3594-3603.

Shaner NC, Campbell RE, Steinbach PA, Giepmans BN, Palmer AE, Tsien RY (2004) Improved monomeric red, orange and yellow fluorescent proteins derived from Discosoma sp. red fluorescent protein. Nat Biotechnol 22:1567-1572.

Smear MC, Tao HW, Staub W, Orger MB, Gosse NJ, Liu Y, Takahashi K, Poo MM, Baier H (2007) Vesicular glutamate transport at a central synapse limits the acuity of visual perception in zebrafish. Neuron 53:65-77.

Sørensen JB, Matti U, Wei SH, Nehring RB, Voets T, Ashery U, Binz T, Neher E, Rettig J (2002) The SNARE protein SNAP-25 is linked to fast calcium triggering of exocytosis. Proc Natl Acad Sci U S A 99:1627-1632.

Vislay-Meltzer RL, Kampff AR, Engert F (2006) Spatiotemporal specificity of neuronal activity directs the modification of receptive fields in the developing retinotectal system. Neuron 50:101-114.

White B, Osterwalder T, Keshishian H (2001) Molecular genetic approaches to the targeted suppression of neuronal activity. Curr Biol 11:R1041R1053.

Wiesel TN (1982) The postnatal development of the visual cortex and the influence of environment. Biosci Rep 2:351-377.

Yu CR, Power J, Barnea G, O’Donnell S, Brown HE, Osborne J, Axel R, Gogos JA (2004) Spontaneous neural activity is required for the establishment and maintenance of the olfactory sensory map. Neuron 42:553-566.

Zhang LI, Tao HW, Holt CE, Harris WA, Poo M (1998) A critical window for cooperation and competition among developing retinotectal synapses. Nature 395:37-44.

Zhang LI, Tao HW, Poo M (2000) Visual input induces long-term potentiation of developing retinotectal synapses. Nat Neurosci 3:708-715. 